\title{
Optimal Boussinesq model for shallow-water waves interacting with a microstructure
}

\author{
Josselin Garnier* \\ Laboratoire de Probabilités et Modèles Aléatoires \& Laboratoire Jacques-Louis Lions, Université Paris 7, 2 Place Jussieu, \\ 75251 Paris Cedex 05, France \\ Roberto A. Kraenkel ${ }^{\dagger}$ \\ Instituto de Fisica Teorica-UNESP, R. Pamplona 145, 01405-900 São Paulo, Brazil \\ André Nachbin \\ Instituto de Matemática Pura e Aplicada, Est. D Castorina 110, Jardim Botânico, Rio de Janeiro, RJ 22460-320, Brazil
}

(Received 20 June 2007; published 12 October 2007)

\begin{abstract}
In this paper, we consider the propagation of water waves in a long-wave asymptotic regime, when the bottom topography is periodic on a short length scale. We perform a multiscale asymptotic analysis of the full potential theory model and of a family of reduced Boussinesq systems parametrized by a free parameter that is the depth at which the velocity is evaluated. We obtain explicit expressions for the coefficients of the resulting effective Korteweg-de Vries (KdV) equations. We show that it is possible to choose the free parameter of the reduced model so as to match the $\mathrm{KdV}$ limits of the full and reduced models. Hence the reduced model is optimal regarding the embedded linear weakly dispersive and weakly nonlinear characteristics of the underlying physical problem, which has a microstructure. We also discuss the impact of the rough bottom on the effective wave propagation. In particular, nonlinearity is enhanced and we can distinguish two regimes depending on the period of the bottom where the dispersion is either enhanced or reduced compared to the flat bottom case.
\end{abstract}

DOI: 10.1103/PhysRevE.76.046311

PACS number(s): 47.15.km, 47.35.Fg, 47.11.St, 47.35.Bb

\section{INTRODUCTION}

The surface elevation of a body of fluid with a periodically varying bottom for two-dimensional flows has been the subject of many papers in the past decades. Different asymptotic regimes can be addressed. Nevertheless research efforts have been mostly concentrated in the Bragg resonance regime [1-7], namely a regime where the water wavelength and the bottom wavelength are comparable. Linear and weakly nonlinear (solitary) waves have been addressed in these works. Very little work has been devoted to rapidly varying topographies as well as for bottoms with abrupt slope variations.

The objective of this paper is to describe the wave propagation in a long-wave asymptotic regime, when the bottom topography is periodic on a short length scale and has an arbitrary slope profile. In [8] Rosales and Papanicolaou derived an effective Korteweg-de Vries (KdV) equation by using a multiscale expansion technique on the potential theory formulation of the problem. In [9] Craig et al. give an alternate derivation of the effective $\mathrm{KdV}$, by applying a perturbation technique on the Hamiltonian system for the Euler equations for water waves. In addition, they consider both the two- and three-dimensional cases in their analysis, and they obtain effective Boussinesq equations that describe the motion of bidirectional long waves. However, the coefficients of the effective $\mathrm{KdV}$ equations are not given explicitly in these

\footnotetext{
*garnier@math.jussieu.fr

${ }^{\dagger}$ kraenkel@ift.unesp.br

*nachbin@impa.br
}

works, but they are expressed as averages of solutions of auxiliary problems. The first goal of our paper is to obtain explicit expressions for the coefficients of the effective $\mathrm{KdV}$ equation in the two-dimensional case, which will allow us to discuss the impact of the rough bottom on the wave propagation. Then, having these explicit $\mathrm{KdV}$ equations at hand, we obtain an optimal Boussinesq system for shallow water (long) wave interaction with a microstructure, here the rapidly varying topography.

Recently Nachbin has obtained a terrain-following Boussinesq system that is a weakly nonlinear, weakly dispersive approximation of the potential theory formulation in the presence of a varying bottom [10]. This was obtained through the use of a conformal mapping transformation in order to change the coordinate system. In the new coordinate system abrupt bottom variations of large amplitude can be dealt with. This mapping strategy was also used in [5] for the Bragg resonance case. Moreover this formulation represents a dramatic reduction of the complexity of the problem, since only one spatial terrain-following coordinate is (asymptotically) used and therefore the space dimension is reduced by one compared to the potential theory equations. In this paper, we show that is possible to obtain an effective $\mathrm{KdV}$ equation from this simplified system without solving auxiliary cell problems. This effective $\mathrm{KdV}$ equation has similar properties as those obtained in [8,9] and allows us to consider more general bottom profiles. For example, the profiles can be discontinuous or even multivalued, with the additional advantage that explicit expressions are obtained for the coefficients of the effective $\mathrm{KdV}$ equation. This fact plays an important role in answering our first goal.

As we shall see, the reduced Boussinesq system presented in this paper is parametrized by a free parameter, namely the 
depth at which the velocity is evaluated. The choice of this free parameter is determined by the specification of the physical properties that the reduced model is required to reproduce with accuracy, when compared to the full potential theory model. Different criteria may lead to different optimized values for the free parameter [11-13]. Hence our second goal in this paper is to show that it is possible to choose the free parameter of our family of Boussinesq systems so that the $\mathrm{KdV}$ limit of the reduced model is the same as the $\mathrm{KdV}$ limit of the full potential theory model. Thus the reduced model is optimal regarding the embedded linear weakly dispersive and weakly nonlinear characteristics of the underlying physical problem, in the presence of a microstructure.

The paper is organized as follows. In Sec. II we formulate the full potential theory. The reduced Boussinesq system is presented in Sec. III. In Secs. IV and V we perform a multiscale analysis of the two models in the presence of a periodic bottom and we derive the limit KdV equations. In particular, the optimization of the reduced Boussinesq model is addressed in Sec. VE and the explicit expressions for the coefficients of the effective $\mathrm{KdV}$ equation are discussed in Sec. V F.

\section{EQUATIONS OF MOTION}

We are in a regime where the fluid dynamic problem is governed by the Euler equations with a free surface at the top of the fluid domain $y=\alpha \eta(x, t)$. The wave profile is described by $\eta(x, t)$. Using the fact that the flow is incompressible and irrotational the problem can be recast in terms of a velocity potential $\phi(x, y, t)$, such that the velocity field is given by $(\widetilde{u}, \widetilde{v})=\nabla \phi$. Following Rosales and Papanicolaou [8], the dimensionless nonlinear potential theory equations are

$$
\beta \phi_{x x}+\phi_{y y}=0, \quad \text { in } \quad-\sqrt{\beta} H(x / \varepsilon)<y<\alpha \eta(x, t),
$$

with free surface conditions at $y=\alpha \eta(x, t)$ :

$$
\begin{gathered}
\phi_{t}+\frac{\alpha}{2}\left(\phi_{x}^{2}+\frac{1}{\beta} \phi_{y}^{2}\right)+\eta=0, \\
\eta_{t}+\alpha \phi_{x} \eta_{x}-\frac{1}{\beta} \phi_{y}=0,
\end{gathered}
$$

and a Neumann condition

$$
\frac{\beta}{\varepsilon} H^{\prime}\left(\frac{x}{\varepsilon}\right) \phi_{x}+\phi_{y}=0
$$

along the highly variable topography described by $y=-\sqrt{\beta} H(x / \varepsilon)$. The parameter $\alpha$ is the ratio of the typical wave amplitude over the mean depth. It governs the strength of the nonlinearity. The parameter $\beta$ is the ratio of the squared mean depth over the squared characteristic wavelength of the wave. It governs the strength of the dispersion. Finally the parameter $\varepsilon$ is the ratio of the topographic length scale over the characteristic wavelength. In other work by the authors [14-21] the topography was taken as disordered and modeled by a random process, and therefore the topographic length scale was the correlation length of the random process. Of great interest is the case of solitary waves in the presence of random topographies as considered by different authors $[14,17,19,22]$. In this paper, the bottom has periodic modulations and the topographic length scale can then be defined as the period of the modulations.

In [10] a conformal mapping is used to map the rough channel onto a flat strip. This is the same as changing variables from a Cartesian $x y$-coordinate system to an orthogonal curvilinear one, namely in, say, $\xi \zeta$ variables. This is done in such a way that at the undisturbed free surface $y \equiv 0$ and $\zeta$ $\equiv 0$ coincide. The topography now is along a ( $\zeta \equiv$ const) curve. Since we are dealing with harmonic functions (through the potential and the conformal map) it easily follows that in the curvilinear coordinates we have a new set of orthogonal velocity components $(u, v)=\nabla \phi(\xi, \zeta, t)$, where $u(\xi, \zeta, t)$ is a terrain-following component, namely tangent to the $\zeta$-level curves. Now the gradient is to be considered in the new set of variables. As shown in [18], in the limit $\alpha \ll 1, \beta \ll 1$, one obtains a family of Boussinesq systems for the wave elevation $\eta$ and the velocity component $u$ that are parametrized by the depth $\zeta$ at which the $u$-velocity component is evaluated. The dispersion relations of these Boussinesq systems depend on this parameter, which therefore change in different interesting ways through a family of Padé approximations from the original potential theory dispersion relation. The choice of this parameter depends on the criterion used to evaluate the quality of the reduced model compared to the full potential theory. This issue is very important from the physical oceanography point of view, as for example shown in [18] regarding a waveform inversion procedure. Namely, a time-reversal procedure is used in order to find, say, a tsunami's initial waveform through the recompression of scattered data near the coast. As shown in [18] the initial amplitude, regarding the tsunami's waveform inversion, is underestimated by the reduced model unless the optimal parameter is used. In [11,12] different strategies are used to optimize some prescribed linear and nonlinear physical characteristics of the reduced model, such as the phase velocity for a given waveband, the linear shoaling characteristics, or the nonlinear transfer of energy between harmonics. To the best of our knowledge, this has only been done for smoothly varying bottoms and shoaling scenarios. Here we extend this type of model optimization to rapidly varying topographies. As in $[11,12]$ we use a free parameter, namely the arbitrary depth parameter for the velocity variable, in order to optimize the scattering properties of solitary waves. This is done by matching the leading order $\mathrm{KdV}$ equations arising from our reduced, variable coefficient, Boussinesq system with that arising from the potential theory framework. At the end we obtain an optimal Boussinesq system for practical applications where a weakly nonlinear wave, such as a solitary wave, interacts with rapidly varying topographic features.

\section{TERRAIN-FOLLOWING BOUSSINESQ SYSTEM}

In this section, we present our reduced model that has the form of a one-parameter family of Boussinesq equations that 
describe the evolution of surface waves in shallow channels with arbitrary, rough topographies [10,18,23]:

$$
\begin{gathered}
M \eta_{t}+\left[\left(1+\frac{\alpha \eta}{M}\right) u\right]_{\xi}-\frac{\beta}{2}\left(y_{0}^{2}-\frac{1}{3}\right)[M \eta]_{\xi \xi t}=0, \\
u_{t}+\eta_{\xi}+\alpha\left[\frac{u^{2}}{2 M^{2}}\right]_{\xi}+\frac{\beta}{2}\left(y_{0}^{2}-1\right) u_{\xi \xi t}=0,
\end{gathered}
$$

where $u$ is the terrain-following velocity evaluated at the relative depth $y_{0}\left(y_{0}=0\right.$ is the bottom, $y_{0}=1$ is the free surface) and $\eta$ is the wave elevation. $\xi$ and $t$ are the space and time coordinates. As mentioned above, system (1) and (2) is derived from the full potential theory equations, in curvilinear coordinates, in the asymptotics $\alpha \ll 1$ and $\beta \ll 1$, and it neglects terms of order $O\left(\alpha^{2}\right), O(\alpha \beta)$, and $O\left(\beta^{2}\right)$. The system is a weakly nonlinear, weakly dispersive approximation of the potential theory equations for an irrotational, incompressible, and inviscid fluid. It is of interest to point out that when $\varepsilon \rightarrow 0$ the wavelength is increasing with respect to the microscale and an averaging process takes place. Rather than calling this a singular limit, we refer the reader to [17] where it was shown that the change to curvilinear coordinates preconditions the equations in the $\varepsilon \rightarrow 0$ limit. Namely the eigenvalue band of the underlying linear $(\alpha=0)$ system remains bounded in opposition to the Cartesian coordinates case where the same linear analysis leads to an ever growing interval containing the linear spectrum. Numerical experiments that corroborate with this fact are presented in $[17,18]$. In particular in [18] random, multiply scattered signals produced by both the Boussinesq and the complete linear potential theory models are compared. An excellent agreement is observed when the depth parameter is optimized numerically. Here this optimal value will be found theoretically through a weakly nonlinear multiscale analysis. Having this in mind we keep the conformal mapping setup and work towards one of our objectives: Similarly to $[11,12]$ we compute an optimal value for $y_{0}$ so that the reduced model retains certain physical properties from the full Euler equations.

The variable coefficient $M(\xi)$ is a smooth topographydependent function which appears as a consequence of our change of variables from cartesian to curvilinear coordinates. The Jacobian for the conformal mapping transformation is defined as $|J|=y_{\xi}^{2}+y_{\zeta}^{2}$ where the Cauchy-Riemann equations have been used. For weakly nonlinear waves, hence to leading order in $\alpha$, the Jacobian along the free surface can be approximated as $|J| \approx y_{\zeta}^{2}(\xi, \zeta=0) \equiv M^{2}(\xi) \quad[10]$. Therefore, $M(\xi)$ is called the metric term. It is computed directly from the physical topography function $H(x / \varepsilon)$ which describes the channel depth. The averaged depth has been normalized to 1 . In the derivation of Eqs. (1) and (2) the topography profile

$$
H(x / \varepsilon)=1+n(x / \varepsilon)
$$

can be rapidly varying, discontinuous, or even multivalued, and no mild slope condition is required. The only requirement is that there exists a constant $C \in(0,1)$ such that $\|n\|_{\infty}$ $\leqslant C$, which simply means that the bottom never goes above the free surface. In the terrain-following system the physical

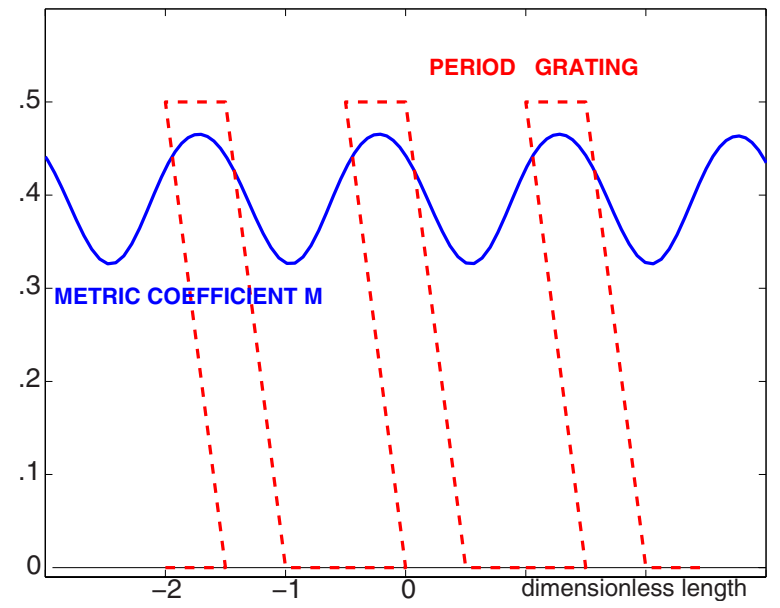

FIG. 1. (Color online) Grating amplitude vs dimensionless coordinate $x$ : A slanted period grating (dashed) and the corresponding periodic metric coefficient (solid) in the $\sqrt{\beta}=1$ regime. Only three components of the grating with period $3 / 2$ are shown for simplicity.

topography $H(x / \varepsilon)=1+n(x / \varepsilon)$ is replaced by the metric coefficient [24]

$$
M(\xi)=1+\frac{\pi}{4 \sqrt{\beta}} \int_{-\infty}^{\infty} \frac{n\left[x\left(\xi_{0},-\sqrt{\beta}\right) / \varepsilon\right]}{\cosh ^{2}\left[\frac{\pi}{2 \sqrt{\beta}}\left(\xi_{0}-\xi\right)\right]} d \xi_{0},
$$

where $(x, y) \mapsto(\xi, \zeta)$ represents the, conformal map, coordinate transformation such that $M(\xi)=y_{\zeta}(\xi, 0)$ [10].

In this paper, we consider that $n$ is a zero-mean periodic function. The periodic orography $H(x / \varepsilon)=1+n(x / \varepsilon)$ is transformed through Eq. (3) into a periodic metric coefficient $M(\xi)$, which is a smoothed version of the original profile. The smoothing is all the more important as $\beta$ is larger. In Sec. V we will perform a detailed analysis of the metric coefficient in the case in which the orography is sinusoidal. Here we briefly show by some illustrations that this transformation allows us to consider complicated, noncontinuous, multivalued profiles. We use Driscoll's Toolbox [25] to perform the numerical Schwarz-Christoffel mapping. In Figs. 1 and 2 we have a slanted grating, of amplitude half the total depth, in two different regimes for the parameter $\beta$. These are to illustrate that the smaller the $\beta$ more details from $n(x / \varepsilon)$ are captured in the metric coefficient $M(\xi)$.

In this paper, we assume that the period of the bottom fluctuations is small compared to the typical wavelength of the wave. This means that we assume that $\varepsilon$ is a small dimensionless parameter. We also assume that the small nonlinearity and dispersion parameters can be written as

$$
\alpha=\varepsilon^{2} \alpha_{0}, \quad \beta=\varepsilon^{2} \beta_{0} .
$$

Here $\alpha_{0}\left(\beta_{0}\right)$ is the normalized nonlinearity (dispersion) parameter which is a nonnegative number of order one. The fact that $\beta$ is of order $\varepsilon^{2}$ ensures that the convolution in Eq. (3) occurs at the same length scale as the period of the fluctuations, which represents the most interesting case. Note that the kernel is of total mass one, hence tending to a Dirac delta function as $\beta \rightarrow 0$. The fact that $\alpha$ is of order $\varepsilon^{2}$ ensures 


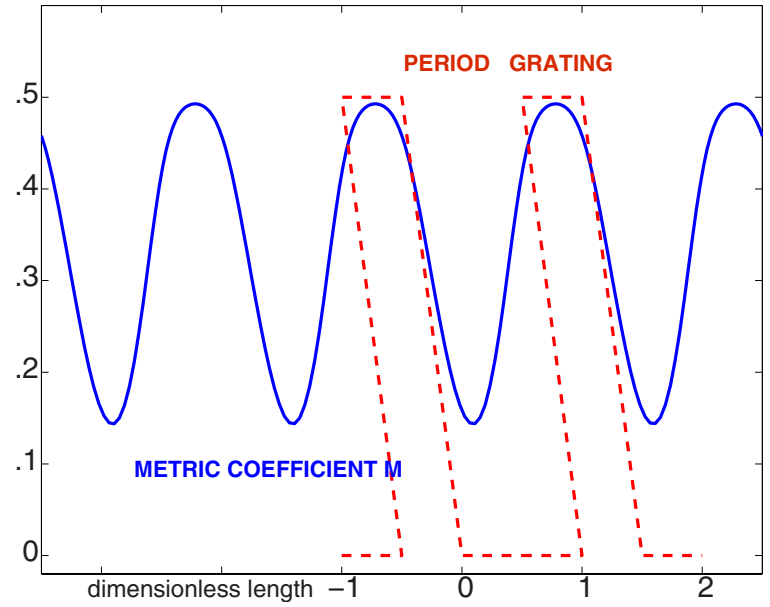

FIG. 2. (Color online) Grating amplitude versus dimensionless coordinate $x$ : Same slanted period grating (dashed) as in Fig. 1 and the corresponding periodic metric coefficient (solid) in the $\sqrt{\beta}=0.5$ regime. Only two components of the grating with period $3 / 2$ are shown.

that the nonlinear and dispersive effects are of the same order so that a nontrivial interplay between nonlinearity, dispersion, and periodic forcing can be expected. This regime is appropriate for the existence of solitary waves and hence also appropriate for the $\mathrm{KdV}$ parameter matching we will perform in optimizing the above Boussinesq system. In the next sections, we perform multiscale asymptotics with both the full potential theory model and the reduced Boussinesq system. We have two goals. The first one is to obtain explicit expressions for the coefficients of the effective $\mathrm{KdV}$ equation in the special case of sinusoidal topography, which will allow us to discuss the impact of the a rough bottom on wave propagation. The second one is to show that it is possible to choose the depth parameter $y_{0}$ in order to match the KdV limit of the full potential theory model and that of the Boussinesq system.

\section{MULTISCALE EXPANSION FOR THE BOUSSINESQ SYSTEM WITH A PERIODIC TOPOGRAPHY}

In this section, we assume that the metric coefficient $M(\xi)$ is a rapidly-varying periodic function of the form

$$
M(\xi)=m\left(\frac{\xi}{\varepsilon}\right),
$$

where $m(s)$ is a smooth periodic function in $s$. In Sec. V we shall obtain the explicit expression of the metric coefficient $m$ in the case in which the physical coefficient $n$ is sinusoidal. Here we consider the asymptotic behavior of the solution $(\eta, u)$ of Eqs. (1) and (2) as $\varepsilon \rightarrow 0$ by analyzing the multiscale expansion of the solution. We will see that the effective behavior for times of order one is described by a standard homogeneous wave equation. For times of order $\varepsilon^{-2}$ we will obtain an effective system of partial differential equations involving dispersion, nonlinearity, and periodic forcing.

\section{A. Propagation times of order one}

We look for an expansion of the form

$$
\begin{aligned}
& \eta(t, \xi)=\eta_{0}\left(t, \xi, \frac{\xi}{\varepsilon}\right)+\varepsilon \eta_{1}\left(t, \xi, \frac{\xi}{\varepsilon}\right)+\cdots, \\
& u(t, \xi)=u_{0}\left(t, \xi, \frac{\xi}{\varepsilon}\right)+\varepsilon u_{1}\left(t, \xi, \frac{\xi}{\varepsilon}\right)+\cdots,
\end{aligned}
$$

where $\eta_{j}$ and $u_{j}$ are periodic in $s=\xi / \varepsilon$ and the averages of $\eta_{1}$ and $u_{1}$ with respect to $s$ are zero. Substituting into Eqs. (1) and (2) and collecting the terms with the same powers in $\varepsilon$, we obtain at order $\varepsilon^{-1}$ :

$$
u_{0_{s}}=0, \quad \eta_{0_{s}}=0,
$$

which shows that the leading-order terms $\left(\eta_{0}, u_{0}\right)$ do not depend on $s=\xi / \varepsilon$. At order $\varepsilon^{0}$, we get

$$
\begin{gathered}
m \eta_{0_{t}}+u_{1_{s}}+u_{0_{\xi}}-\frac{\beta_{0}}{2}\left(y_{0}^{2}-\frac{1}{3}\right) m_{s s} \eta_{0_{t}}=0, \\
u_{0_{t}}+\eta_{1_{s}}+\eta_{0_{\xi}}=0 .
\end{gathered}
$$

By taking the average with respect to $s$, we obtain the compatibility equations:

$$
\begin{gathered}
\langle m\rangle \eta_{0_{t}}+u_{0_{\xi}}=0, \\
u_{0_{t}}+\eta_{0_{\xi}}=0,
\end{gathered}
$$

where $\langle\cdot\rangle$ stands for an average in $s$. This shows that $\left(\eta_{0}, u_{0}\right)$ obeys a standard wave equation with the effective velocity $v=\langle m\rangle^{-1 / 2}$ in the $(t, \xi)$ variables. We can introduce the leftand rightgoing modes $A$ and $B$,

$$
A=\langle m\rangle^{1 / 4} \eta_{0}+\langle m\rangle^{-1 / 4} u_{0}, \quad B=\langle m\rangle^{1 / 4} \eta_{0}-\langle m\rangle^{-1 / 4} u_{0} .
$$

They satisfy the two uncoupled transport equations

$$
A_{t}+v A_{\xi}=0, \quad B_{t}-v B_{\xi}=0,
$$

and they are given by

$$
A(t, \xi)=A(t=0, \xi-v t), \quad B(t, \xi)=B(t=0, \xi+v t) .
$$

Consequently, an arbitrary initial condition gives rise to two waves $A$ and $B$, one propagating to the right with velocity $+v$ and one propagating to the left with velocity $-v$. For instance, an initial condition such that $u_{0}(t=0, \xi)$ $=\langle m\rangle^{1 / 2} \eta_{0}(t=0, \xi)=\langle m\rangle^{1 / 4} f(\xi)$ generates a pure right-going wave, that propagates without distortion for times of order one:

$$
u_{0}(t, \xi)=\langle m\rangle^{-1 / 4} f(\xi-v t), \quad \eta_{0}(t, \xi)=\langle m\rangle^{1 / 4} f(\xi-v t) .
$$

This result holds true for times of order $O(1)$.

\section{B. Propagation times of order $\varepsilon^{-2}$}

We investigate the slow evolution of the solution $(\eta, u)$ for times of order $\varepsilon^{-2}$ in the frame moving with the velocity $v$. We look for an expansion of the form 


$$
\begin{aligned}
\eta(t, \xi)= & \eta_{0}\left(\xi-v t, \varepsilon^{2} t\right)+\varepsilon \eta_{1}\left(\xi-v t, \frac{\xi}{\varepsilon}, \varepsilon^{2} t\right) \\
& +\varepsilon^{2} \eta_{2}\left(\xi-v t, \frac{\xi}{\varepsilon}, \varepsilon^{2} t\right)+\cdots, \\
u(t, \xi)= & u_{0}\left(\xi-v t, \varepsilon^{2} t\right)+\varepsilon u_{1}\left(\xi-v t, \frac{\xi}{\varepsilon}, \varepsilon^{2} t\right) \\
& +\varepsilon^{2} u_{2}\left(\xi-v t, \frac{\xi}{\varepsilon}, \varepsilon^{2} t\right)+\cdots,
\end{aligned}
$$

where $\eta_{j}$ and $u_{j}$ are periodic in $s=\xi / \varepsilon$ and admit slow variations in $\tau=\varepsilon^{2} t$. We denote $\chi=\xi-v t$. At order $\varepsilon^{0}$ we find that

$$
\begin{gathered}
-v m \eta_{0_{t}}+u_{1_{s}}+u_{0_{\chi}}+v \frac{\beta_{0}}{2}\left(y_{0}^{2}-\frac{1}{3}\right) m_{s s} \eta_{0_{\chi}}=0, \\
-v u_{0_{\chi}}+\eta_{1_{s}}+\eta_{0_{\chi}}=0 .
\end{gathered}
$$

By taking the average in $s$, we get that $v^{2}\langle m\rangle=1$, which is satisfied, and

$$
\eta_{0}=v u_{0},
$$

which means that the leading-order terms have the form of a rightgoing wave. Hence, as expected, no reflection is generated by the homogenized bottom. Besides the corrections $\left(\eta_{1}, u_{1}\right)$ are given by

$$
u_{1}=v\left[m_{a}-m_{s} \frac{\beta_{0}}{2}\left(y_{0}^{2}-\frac{1}{3}\right)\right] \eta_{0_{\chi}}+\bar{u}_{1}, \quad \eta_{1}=\bar{\eta}_{1},
$$

where $\left(\bar{u}_{1}, \bar{\eta}_{1}\right)$ do not depend on $s$ and $m_{a}$ is the zero-mean periodic function

$$
m_{a}(s)=\tilde{m}_{a}(s)-\left\langle\tilde{m}_{a}\right\rangle, \quad \tilde{m}_{a}(s)=\int_{0}^{s} m(s)-\langle m\rangle d s .
$$

Note that $\left\langle u_{1}\right\rangle=\bar{u}_{1}$ and $\left\langle\eta_{1}\right\rangle=\bar{\eta}_{1}$.

At order $\varepsilon$ we obtain

$$
\begin{aligned}
& -v m \eta_{1_{\chi}}+u_{2_{s}}+u_{1_{\chi}}+\alpha_{0} u_{0} \eta_{0}\left(\frac{1}{m}\right)_{s}+v \frac{\beta_{0}}{2}\left(y_{0}^{2}-\frac{1}{3}\right)\left[m \eta_{1_{\chi}}\right]_{s s} \\
& \quad=0 \\
& -v u_{1_{\chi}}+\eta_{2_{s}}+\eta_{1_{\chi}}+\frac{\alpha_{0} u_{0}^{2}}{2}\left(\frac{1}{m^{2}}\right)_{s}-\frac{\beta_{0}}{2}\left(y_{0}^{2}-1\right) u_{1_{\chi s s}}=0 .
\end{aligned}
$$

By taking the average in $s$, we obtain the compatibility equations

$$
\begin{gathered}
-v\langle m\rangle \bar{\eta}_{1_{\chi}}+\bar{u}_{1_{\chi}}=0, \\
-v \bar{u}_{1_{\chi}}+\bar{\eta}_{1_{\chi}}=0,
\end{gathered}
$$

which are satisfied if

$$
\bar{\eta}_{1}=v \bar{u}_{1} .
$$

Besides, the corrections $\left(\eta_{2}, u_{2}\right)$ have the form

$$
\begin{aligned}
\eta_{2}= & v^{2}\left(m_{b}-\frac{\beta_{0}}{3}(m-\langle m\rangle)\right) \eta_{0_{\chi x}}-\frac{\beta_{0}^{2}}{4}\left(y_{0}^{2}-\frac{1}{3}\right) \\
& \times\left(y_{0}^{2}-1\right) m_{s s} \eta_{0_{\chi x}}-\frac{\alpha_{0} \eta_{0}^{2}}{2 v^{2}}\left(\frac{1}{m^{2}}-\left\langle\frac{1}{m^{2}}\right\rangle\right)+\bar{\eta}_{2}, \\
u_{2}= & v\left(-m_{b}+\frac{\beta_{0}}{2}\left(y_{0}^{2}-\frac{1}{3}\right)(m-\langle m\rangle)\right) \eta_{0 \chi x} \\
- & \frac{\alpha_{0} \eta_{0}^{2}}{v}\left(\frac{1}{m}-\left\langle\frac{1}{m}\right\rangle\right)+v\left[m_{a}-\frac{\beta_{0}}{2}\left(y_{0}^{2}-\frac{1}{3}\right) m_{s}\right] \bar{\eta}_{1_{\chi}}+\bar{u}_{2},
\end{aligned}
$$

where $\left(\bar{\eta}_{2}, \bar{u}_{2}\right)$ do not depend on $s$ and $m_{b}$ is the zero-mean periodic function

$$
m_{b}(s)=\widetilde{m}_{b}(s)-\left\langle\tilde{m}_{b}\right\rangle, \quad \tilde{m}_{b}(s)=\int_{0}^{s} m_{a}(s) d s .
$$

Note once again that $\left\langle u_{2}\right\rangle=\bar{u}_{2}$ and $\left\langle\eta_{2}\right\rangle=\bar{\eta}_{2}$. In the following, we denote $\widetilde{\eta}_{2}=\eta_{2}-\bar{\eta}_{2}$.

At order $\varepsilon^{2}$ we obtain the system

$$
\begin{gathered}
m\left(\eta_{0_{\tau}}-v \eta_{2_{\chi}}\right)+u_{3_{s}}+u_{2_{\chi}}+\alpha_{0}\left(\frac{1}{m}\left(\eta_{1} u_{0}+\eta_{0} u_{1}\right)\right)_{s} \\
+\alpha_{0}\left(u_{0} \eta_{0}\right)_{\chi} \frac{1}{m}-\frac{\beta_{0}}{2}\left(y_{0}^{2}-\frac{1}{3}\right)\left[m\left(\eta_{0_{\tau}}-v \eta_{2_{\chi}}\right)\right]_{s s} \\
+\frac{\beta_{0}}{2}\left(y_{0}^{2}-\frac{1}{3}\right)\left\{2 v\left[m \eta_{1_{\chi \chi}}\right]_{s}+v m \eta_{0_{\chi \chi \chi}}\right\}=0,
\end{gathered}
$$

$$
\begin{aligned}
u_{0}- & v u_{2_{\chi}}+\eta_{3_{s}}+\eta_{2_{\chi}}+\alpha_{0} u_{0}\left(\frac{u_{1}}{m^{2}}\right)_{s}+\frac{\alpha_{0}\left(u_{0}^{2}\right)_{\chi}}{2 m^{2}}-v \frac{\beta_{0}}{2}\left(y_{0}^{2}-1\right) \\
& \times\left(u_{0_{\chi \chi \chi}}+2 u_{1_{\chi \chi s}}+u_{2_{\chi s s}}\right)=0 .
\end{aligned}
$$

By taking the average with respect to $s$, we get the compatibility equations

$$
\begin{gathered}
\langle m\rangle \eta_{0_{\tau}}-v\left\langle m \tilde{\eta}_{2_{\chi}}\right\rangle-v\langle m\rangle \bar{\eta}_{2_{\chi}}+\bar{u}_{2_{\chi}}+\frac{\alpha_{0}}{v}\left(\eta_{0}^{2}\right)_{\chi}\left\langle\frac{1}{m}\right\rangle \\
+v \frac{\beta_{0}\langle m\rangle}{2}\left(y_{0}^{2}-\frac{1}{3}\right) \eta_{0_{\chi \chi \chi}}=0, \\
\frac{1}{v} \eta_{0_{\tau}}-v \bar{u}_{2_{\chi}}+\bar{\eta}_{2_{\chi}}+\left\langle\frac{1}{m^{2}}\right\rangle \frac{\alpha_{0}\left(\eta_{0}^{2}\right)_{\chi}}{2 v^{2}}-\frac{\beta_{0}}{2}\left(y_{0}^{2}-1\right) \eta_{0_{\chi \chi \chi}}=0 .
\end{gathered}
$$

We multiply by $v$ the first equation and add the second equation, which gives

$$
\frac{2}{v} \eta_{0_{\tau}}-v^{2}\left\langle m \tilde{\eta}_{2_{\chi}}\right\rangle+\alpha_{0}\left(\eta_{0}^{2}\right)_{\chi}\left(\left\langle\frac{1}{m}\right\rangle+\frac{1}{2}\left\langle\frac{1}{m^{2}}\right\rangle\langle m\rangle\right)+\frac{\beta_{0}}{3} \eta_{0_{\chi \chi \chi}}
$$$$
=0 \text {. }
$$

Using the expression of $\widetilde{\eta}_{2}$ and the integration by parts formulas $\left\langle m m_{b}\right\rangle=-\left\langle m_{a}^{2}\right\rangle$ and $\left\langle m m_{s s}\right\rangle=-\left\langle m_{s}^{2}\right\rangle$ : 


$$
\begin{aligned}
\left\langle m \tilde{\eta}_{2}\right\rangle= & v^{2}\left[-\left\langle m_{a}^{2}\right\rangle-\frac{\beta_{0}}{3}\left\langle(m-\langle m\rangle)^{2}\right\rangle+\frac{\beta_{0}^{2}}{4}\left(y_{0}^{2}-\frac{1}{3}\right)\left(y_{0}^{2}-1\right)\right. \\
& \left.\times\left\langle m_{s}^{2}\right\rangle\right] \eta_{0_{\chi \chi \chi}}-\frac{\alpha_{0}\left(\eta_{0}^{2}\right)_{\chi}}{2 v^{2}}\left(\left\langle\frac{1}{m}\right\rangle-\left\langle\frac{1}{m^{2}}\right\rangle\langle m\rangle\right),
\end{aligned}
$$

we obtain the effective equation for $\eta_{0}$ :

$$
\begin{aligned}
2\langle m\rangle^{1 / 2} \eta_{0_{\tau}}+\frac{3 \alpha_{0}}{2}\left(\eta_{0}^{2}\right)_{\chi}\left\langle\frac{1}{m}\right\rangle+\left[\frac{\beta_{0}}{3}+\frac{\left\langle m_{a}^{2}\right\rangle}{\langle m\rangle^{2}}\right. \\
\left.+\frac{\beta_{0}}{3} \frac{\left\langle(m-\langle m\rangle)^{2}\right\rangle}{\langle m\rangle^{2}}-\frac{\beta_{0}^{2}}{4}\left(y_{0}^{2}-\frac{1}{3}\right)\left(y_{0}^{2}-1\right) \frac{\left\langle m_{s}^{2}\right\rangle}{\langle m\rangle^{2}}\right] \eta_{{ }_{\chi \chi \chi}}=0 .
\end{aligned}
$$

This system is expressed in the variables $(\tau, \chi)$, where $\tau$ $=\varepsilon^{2} t$ is the slow time variable and $\chi$ is the traveling coordinate moving with velocity $v: \chi=\xi-v t$. The metric coefficient $M(\xi)$ is defined by $M(\xi)=y_{\zeta}(\xi, 0)$, which is also equal to $x_{\xi}(\xi, 0)$ by the Cauchy-Riemann equation. Therefore, at the undisturbed surface,

$$
\frac{d x}{d \xi}(\xi, 0)=M(\xi)
$$

This equation allows us to state the system in the original variables $(x, t)$. Indeed, $M$ is a rapidly varying periodic function with mean $\langle M\rangle=1+\langle m\rangle$, so that the effective equation for $\eta_{0}$ can be rewritten as the $\mathrm{KdV}$ equation

$$
\eta_{0}+\frac{3 \alpha^{*}}{4}\left(\eta_{0}^{2}\right)_{X}+\frac{\beta^{*}}{6} \eta_{0_{X X X}}=0
$$

where $X=x-v^{*} t$ is the spatial coordinate in the frame moving with the velocity

$$
v^{*}=\langle m\rangle^{1 / 2}
$$

and the nonlinearity and dispersion parameters are given explicitly by

$$
\begin{gathered}
\alpha^{*}=\alpha_{0}\left\langle\frac{1}{m}\right\rangle\langle m\rangle^{1 / 2}, \\
\beta^{*}=\beta_{0}\langle m\rangle^{5 / 2}\left[1+\frac{3\left\langle m_{a}^{2}\right\rangle}{\beta_{0}\langle m\rangle^{2}}+\frac{\left\langle(m-\langle m\rangle)^{2}\right\rangle}{\langle m\rangle^{2}}\right. \\
\left.-\frac{3 \beta_{0}}{4}\left(y_{0}^{2}-\frac{1}{3}\right)\left(y_{0}^{2}-1\right) \frac{\left\langle m_{s}^{2}\right\rangle}{\langle m\rangle^{2}}\right] .
\end{gathered}
$$

The signs of the corrective terms can be discussed.

(i) As first noted in [8] and proved in [9], the effective velocity is strictly smaller than one as soon as $n(x)$ is periodic and nonidentically zero. This means that the effective depth is reduced and that the metric coefficient satisfies $\langle m\rangle \leqslant 1$.

(ii) The expression (13) allows us to claim that nonlinearity is enhanced. Indeed, by Cauchy-Schwarz inequality, we obtain

$$
1=\left\langle m^{1 / 2} m^{-1 / 2}\right\rangle^{2} \leqslant\langle m\rangle\left\langle\frac{1}{m}\right\rangle=\langle m\rangle^{1 / 2}\langle m\rangle^{1 / 2}\left\langle\frac{1}{m}\right\rangle
$$

and therefore

$$
1 \leqslant\langle m\rangle^{1 / 2}\left\langle\frac{1}{m}\right\rangle=\frac{\alpha^{*}}{\alpha_{0}},
$$

where we have used the fact that $\langle m\rangle \leqslant 1$ once again.

(iii) The sign of the dispersive correction $\beta^{*}-\beta_{0}$ cannot be determined without specifying the bottom topography modulation. As we shall see in the next section, dispersion can be enhanced or reduced depending on the period of the modulation.

We recall that the effective parameters in $[8,9]$ are defined implicitly through the solution of auxiliary cell problems. Below we present an example where we compute all parameters analytically. This is a useful example for validating the capabilities of the present (reduced) Boussinesq model in comparison with potential theory and also for discussing quantitatively the impact of the periodic modulations of the bottom on wave propagation. Moreover, the example presented below will allow us to compute explicitly the optimal value for the depth parameter $y_{0}$, so that the above $\mathrm{KdV}$ equations matches the one that arises directly from the potential theory model.

\section{MULTISCALE EXPANSION FOR A SINUSOIDAL OROGRAPHY}

In this section, we consider that $n(x)$ is the periodic sinusoidal function

$$
n(x)=n_{1} \sin (k x)
$$

with $n_{1} \in(0,1)$ and $k>0$.

\section{A. Asymptotic analysis of the conformal mapping}

The metric coefficient $M(\xi)$ is defined by Eq. (3). It is given by the convolution of a smooth explicit kernel with the composition of the periodic function $n$ and the real part of the conformal map $x(\xi, \zeta)$ evaluated at the unperturbed bottom $-\sqrt{\beta}=-\sqrt{\beta_{0}} \varepsilon$. The goal of this subsection is to get an asymptotic expansion of $x\left(\xi,-\sqrt{\beta_{0}} \varepsilon\right)$ as $\varepsilon \rightarrow 0$. This requires are to analyze the conformal mapping introduced in $[10,21]$. We start by considering the Neumann problem

$$
\Delta \xi(x, y)=0
$$

with the boundary condition $\xi_{y}=0$ at $y=0$ and

$$
\xi_{n}=\xi_{y}+\sqrt{\beta_{0}} n^{\prime}(x / \varepsilon) \xi_{x}=0 \quad \text { at } \quad y=-\sqrt{\beta_{0}} \varepsilon[1+n(x / \varepsilon)] .
$$

To solve this problem in the asymptotic $\varepsilon \rightarrow 0$ regime, we first replace the boundary condition at the random bottom $y=-\sqrt{\beta_{0}} \varepsilon[1+n(x / \varepsilon)]$ by a boundary condition at the flat bottom $y=-\sqrt{\beta_{0}} \varepsilon$ :

$$
\xi_{y}+\sqrt{\beta_{0}} n_{1} k \cos \left(\frac{k x}{\varepsilon}\right) \xi_{x}=R_{1}^{\varepsilon}+R_{2}^{\varepsilon},
$$

with 


$$
\begin{aligned}
R_{1}^{\varepsilon}= & \sqrt{\beta_{0}} \varepsilon n_{1} \sin \left(\frac{k x}{\varepsilon}\right) \\
& \times \int_{0}^{1} \xi_{y y}\left\{x,-\sqrt{\beta_{0}} \varepsilon\left[1+\theta n_{1} \sin \left(\frac{k x}{\varepsilon}\right)\right]\right\} d \theta, \\
R_{2}^{\varepsilon}= & \beta_{0} \varepsilon n_{1}^{2} k \sin \left(\frac{k x}{\varepsilon}\right) \cos \left(\frac{k x}{\varepsilon}\right) \\
& \times \int_{0}^{1} \xi_{x y}\left\{x,-\sqrt{\beta_{0}} \varepsilon\left[1+\theta n_{1} \sin \left(\frac{k x}{\varepsilon}\right)\right]\right\} d \theta .
\end{aligned}
$$

The corrective terms $R_{1}^{\varepsilon}$ and $R_{2}^{\varepsilon}$ are the Lagrange remainders of the Taylor expansions of $\xi_{y}$ and $\sqrt{\beta_{0}} \cos (k x / \varepsilon) \xi_{x}$ at $y=-\sqrt{\beta_{0}} \varepsilon$. The solution has the form

$$
\xi(x, y)=\xi_{0}(x)+\xi_{1}(x, y)+\xi_{r}(x, y),
$$

where $\xi_{0}(x)=x$ is the identity describing the conformal map in absence of a perturbation. Then $\xi_{1}$ is the first order corrective term satisfying the Laplace equation $\Delta \xi_{1}=0$, the boundary condition $\xi_{1}=0$ at $y=0$ and the boundary condition $\xi_{1}+\sqrt{\beta_{0}} n_{1} k \cos (k x / \varepsilon) \xi_{0_{x}}=0$ at $y=-\sqrt{\beta_{0}} \varepsilon$. The solution is

$$
\xi_{1}(x, y)=\frac{\sqrt{\beta_{0}} n_{1} \varepsilon}{\sinh \left(k \sqrt{\beta_{0}}\right)} \cosh \left(\frac{k y}{\varepsilon}\right) \cos \left(\frac{k x}{\varepsilon}\right) .
$$

At the unperturbed bottom $y=-\sqrt{\beta_{0}} \varepsilon$ the correction $\xi_{1}$ has the form

$$
\xi_{1}\left(x,-\sqrt{\beta_{0}} \varepsilon\right)=\frac{\sqrt{\beta_{0}} n_{1} \varepsilon}{\tanh \left(k \sqrt{\beta_{0}}\right)} \cos \left(\frac{k x}{\varepsilon}\right) .
$$

This result also shows that $R_{1}^{\varepsilon}=O\left(n_{1}^{2}\right)$ and $R_{2}^{\varepsilon}=O\left(n_{1}^{3}\right)$ which means that the terms $R_{j}^{\varepsilon}$ are higher-order corrections in Eq. (16) in the case $n_{1} \ll 1$. Inverting relation (17) at the undisturbed bottom, we have

$$
x\left(\xi,-\sqrt{\beta_{0}} \varepsilon\right)=\xi-\varepsilon n_{1} \frac{\sqrt{\beta_{0}}}{\tanh \left(k \sqrt{\beta_{0}}\right)} \cos \left(\frac{k \xi}{\varepsilon}\right)+O\left(\varepsilon n_{1}^{2}\right) .
$$

\section{B. Expansion of the metric coefficient}

We use the integral expression (3) of $M(\xi)$ and the expansion (19) of $x\left(\xi,-\sqrt{\beta_{0}} \varepsilon\right)$ :

$$
\begin{aligned}
M(\xi)= & 1+\frac{\pi}{4 \sqrt{\beta_{0}} \varepsilon} \int_{-\infty}^{\infty} \frac{n_{1} \sin \left(\frac{k x\left(\xi_{0},-\varepsilon \sqrt{\beta_{0}}\right)}{\varepsilon}\right)}{\cosh ^{2}\left[\frac{\pi}{2 \sqrt{\beta_{0}} \varepsilon}\left(\xi_{0}-\xi\right)\right]} d \xi_{0} \\
= & 1+\frac{\pi n_{1}}{4 \sqrt{\beta_{0}} \varepsilon} \int_{-\infty}^{\infty} \frac{\sin \left(\frac{k \xi_{0}}{\varepsilon}\right)}{\cosh ^{2}\left[\frac{\pi}{2 \sqrt{\beta_{0}} \varepsilon}\left(\xi_{0}-\xi\right)\right]} d \xi_{0} \\
& \left.-\frac{\pi n_{1}^{2} k}{4 \tanh \left(k \sqrt{\beta_{0}}\right) \varepsilon} \int_{-\infty}^{\infty} \frac{\cos ^{2}\left(\frac{k \xi_{0}}{\varepsilon}\right)}{\cosh ^{2}\left[\frac{\pi}{2 \sqrt{\beta_{0}} \varepsilon}\left(\xi_{0}-\xi\right)\right.}\right]^{d \xi_{0}} \\
& +O\left(n_{1}^{3}\right),
\end{aligned}
$$

which gives

$$
\begin{aligned}
M(\xi)= & 1+\frac{n_{1} k \sqrt{\beta_{0}}}{\sinh \left(k \sqrt{\beta_{0}}\right)} \sin \left(\frac{k \xi}{\varepsilon}\right)-\frac{n_{1}^{2} k \sqrt{\beta_{0}}}{2 \tanh \left(k \sqrt{\beta_{0}}\right)} \\
& -\frac{n_{1}^{2} k^{2} \beta_{0}}{2 \sinh ^{2}\left(k \sqrt{\beta_{0}}\right)} \cos \left(\frac{2 k \xi}{\varepsilon}\right)+O\left(n_{1}^{3}\right) .
\end{aligned}
$$

This expansion exhibits several interesting features.

(i) The average physical bottom is one, but the average of the metric coefficient is smaller than one:

$$
\langle M\rangle=1-\frac{n_{1}^{2} k \sqrt{\beta_{0}}}{2 \tanh \left(k \sqrt{\beta_{0}}\right)}+O\left(n_{1}^{3}\right) .
$$

The fact that a zero-mean varying random topography can give rise to a non-zero average depth through the conformal mapping was already observed in the numerical simulations reported in [20] and analyzed in [19] in the case of a random topography. This is reminiscent of the ellipticity of the conformal mapping problem, namely that the effect from one boundary on the other side of the domain decays very rapidly. As a consequence, the surface wave is more sensitive to the peaks of the rough bottom than to the valleys, which gives a bias in the evaluation of the mean.

(ii) The fundamental fluctuations of the metric coefficient are reduced compared to the ones of the topography. The reduction factor is $k \sqrt{\beta_{0}} / \sinh \left(k \sqrt{\beta_{0}}\right)$ which is close to one when $k \sqrt{\beta_{0}}$ is small and very small when $k \sqrt{\beta_{0}}$ is large.

(iii) Second harmonic modulations are generated with an amplitude that is smaller compared to the fundamental modulations. Besides, we can expect the generation of a cascade of harmonics when taking into account all terms of order $n_{1}^{k}$.

(iv) By Eqs. (17) and (18) we have

$$
\frac{d \xi}{d x}(x, 0)=1-\frac{n_{1} k \sqrt{\beta_{0}}}{\sinh \left(k \sqrt{\beta_{0}}\right)} \sin \left(\frac{k x}{\varepsilon}\right)+O\left(n_{1}^{2}\right),
$$

which gives after inversion 


$$
\frac{d x}{d \xi}(\xi, 0)=1+\frac{n_{1} k \sqrt{\beta_{0}}}{\sinh \left(k \sqrt{\beta_{0}}\right)} \sin \left(\frac{k \xi}{\varepsilon}\right)+O\left(n_{1}^{2}\right),
$$

in agreement with Eqs. (10) and (20).

\section{Effective wave propagation}

We can now apply the result of Sec. IV. We introduce the parameters $m_{0}$ and $m_{1}$ :

$$
m_{0}=1-\frac{n_{1}^{2} k \sqrt{\beta_{0}}}{2 \tanh \left(k \sqrt{\beta_{0}}\right)}, \quad m_{1}=\frac{n_{1} k \sqrt{\beta_{0}}}{\sinh \left(k \sqrt{\beta_{0}}\right)} .
$$

The averaged parameters needed to compute the coefficients of the effective $\mathrm{KdV}$ equation (11) are given explicitly by

$$
\begin{gathered}
\langle m\rangle=m_{0}, \quad\left\langle(m-\langle m\rangle)^{2}\right\rangle=\frac{m_{1}^{2}}{2}, \\
\left\langle m_{s}^{2}\right\rangle=\frac{k^{2} m_{1}^{2}}{2}, \quad\left\langle m_{a}^{2}\right\rangle=\frac{m_{1}^{2}}{2 k^{2}}, \\
\left\langle\frac{1}{m}\right\rangle=\frac{1}{m_{0}}\left(1-\frac{m_{1}^{2}}{m_{0}^{2}}\right)^{-1 / 2}, \\
\left\langle\frac{1}{m^{2}}\right\rangle=\frac{1}{m_{0}^{2}}\left(1-\frac{m_{1}^{2}}{m_{0}^{2}}\right)^{-3 / 2} .
\end{gathered}
$$

By collecting all terms of order $n_{1}^{2}$, we obtain

$$
\begin{gathered}
v^{* 2}=1-\frac{n_{1}^{2}}{2} \frac{\sqrt{\beta_{0}} k}{\tanh \left(\sqrt{\beta_{0}} k\right)}, \\
\frac{\alpha^{*}}{\alpha_{0}}=1+\frac{n_{1}^{2}}{2}\left[\left(\frac{\sqrt{\beta_{0}} k}{\sinh \left(\sqrt{\beta_{0}} k\right)}\right)^{2}+\frac{1}{2} \frac{\sqrt{\beta_{0}} k}{\tanh \left(\sqrt{\beta_{0}} k\right)}\right], \\
\frac{\beta^{*}}{\beta_{0}}=1+\frac{n_{1}^{2}}{2}\left\{\left[\frac{3}{\beta_{0} k^{2}}+1-\frac{3 \beta_{0} k^{2}}{4}\left(y_{0}^{2}-\frac{1}{3}\right)\left(y_{0}^{2}-1\right)\right]\right. \\
\left.\times\left(\frac{\sqrt{\beta_{0}} k}{\sinh \left(\sqrt{\beta_{0}} k\right)}\right)^{2}-\frac{5}{2} \frac{\sqrt{\beta_{0}} k}{\tanh \left(\sqrt{\beta_{0}} k\right)}\right\} .
\end{gathered}
$$

The main features of the effective system (that will be discussed in more detail in Sec. V F) are (i) For a mean-zero topography the velocity is reduced $\left(v^{*}<1\right)$. (ii) The nonlinearity is enhanced $\left(\alpha^{*}>\alpha_{0}\right)$. (iii) The dispersion can be enhanced or reduced, depending on $\beta_{0} k^{2}$.

The dependence of the KdV's dispersion parameter with respect to $y_{0}$ is noticeable. Note that in the flat bottom case $[11,18]$ the depth parameter does not affect the cubic term of the dispersion relation. Nevertheless, here it does affect the cubic term in the presence of a rapidly varying periodic forcing. Namely, the Boussinesq system leads an effective KdV equation whose dispersion relation depends on $y_{0}$. This is very fortunate because it provides us with an optimization procedure for the Boussinesq system, as follows. In the next section, following [8], we will compute the coefficients of the effective $\mathrm{KdV}$ equation given directly by the longwavelength asymptotics of the potential theory in the presence of a rough bottom. We shall then show that the full potential theory model and the reduced Boussinesq model give the same averaged $\mathrm{KdV}$ equation if the parameter $y_{0}$ is chosen in a suitable, hence optimal, way.

\section{Multiscale expansion of the potential flow}

In [8] the authors derive effective equations for the surface elevation of gravity waves with a periodic bottom. They apply a multiscale expansion technique to the potential flow formulation of the problem expressed in the cartesian coordinates. The averaged equation has the form of a KdV equation. The coefficients are not given explicitly, but must be determined by solving auxiliary problems. More precisely, the coefficients of the effective $\mathrm{KdV}$ equation (velocity, nonlinearity, and dispersion coefficients) are expressed as averages of the solutions of cell problems, which are elliptic problems with Neumann boundary conditions in the strip $-1-n(x / \varepsilon) \leqslant y \leqslant 0$. We here reformulate the results in a way that allows comparison with our own results. Besides, we expand the effective coefficients obtained in [8] so that the comparisons are quantitative, and this allows us to identity the value of the parameter $y_{0}$ in our reduced model that fits the results of the potential flow theory.

The elliptic cell problems are defined in terms of the operators:

$$
\begin{gathered}
\mathcal{L}=\partial_{y y}+\beta_{0} \partial_{z z} \text { in }-1-n(z)<y<0, \\
\mathcal{L}_{b}=\partial_{y}+\beta_{0} n^{\prime}(z) \partial_{z} \text { on } y=-1-n(z), \\
\mathcal{L}_{s}=\partial_{y} \text { on } y=0,
\end{gathered}
$$

where $z=x / \varepsilon$. The cell, bottom, and surface averages are defined by

$$
\begin{gathered}
\langle a\rangle=\frac{1}{L} \int_{0}^{L} d z \int_{-1-n(z)}^{0} d y a(z, y), \\
\langle a\rangle_{b}=\frac{1}{L} \int_{0}^{L} d z a[z,-1-n(z)], \\
\langle a\rangle_{s}=\frac{1}{L} \int_{0}^{L} d z a(z, 0),
\end{gathered}
$$

where $L$ is the period of $n(z)$.

First cell problem:

$$
\mathcal{L} A=0, \quad \mathcal{L}_{b} A=-\beta_{0} n^{\prime}(z), \quad \mathcal{L}_{s} A=0,
$$

with periodic dependence in $z$ and cell average equal to zero. If $n(z)=n_{1} \sin (k z)$, then the solution can be expanded in powers of $n_{1}$ : 


$$
\begin{aligned}
A(z, y)= & \sqrt{\beta_{0}} n_{1} \cos (k z) \frac{\cosh \left(k \sqrt{\beta_{0}} y\right)}{\sinh \left(k \sqrt{\beta_{0}}\right)} \\
& -\frac{\beta_{0} n_{1}^{2} k}{4} \sin (2 k z) \frac{\cosh \left(2 k \sqrt{\beta_{0}} y\right)}{\sinh ^{2}\left(k \sqrt{\beta_{0}}\right)}+O\left(n_{1}^{3}\right) .
\end{aligned}
$$

The effective velocity, provided in [8], is given by

$$
v^{\star 2}=1-\left\langle A_{z}^{2}+\beta_{0}^{-1} A_{y}^{2}\right\rangle,
$$

which, by the results above, can be expanded as

$$
v^{\star 2}=1-\frac{n_{1}^{2}}{2} \frac{k \sqrt{\beta_{0}}}{\tanh \left(k \sqrt{\beta_{0}}\right)}+O\left(n_{1}^{3}\right) .
$$

Second cell problem:

$$
\begin{gathered}
\mathcal{L} B=-\beta_{0}\left(2 A_{z}+1\right), \quad \mathcal{L}_{b} B=-\beta_{0} n^{\prime}(z) A, \\
\text { and } \mathcal{L}_{s} B=-\beta_{0} v^{2},
\end{gathered}
$$

with periodic dependence in $z$ and cell average equal to zero. The solution can be expanded in powers of $n_{1}$ :

$$
\begin{aligned}
B(z, y)= & -\beta_{0}\left(\frac{y^{2}}{2}+y+\frac{1}{3}\right)+\beta_{0} n_{1} \sin (k z)\left[\frac{y \sinh \left(k \sqrt{\beta_{0}} y\right)}{\sinh \left(k \sqrt{\beta_{0}}\right)}\right. \\
& \left.-\frac{\cosh \left(k \sqrt{\beta_{0}} y\right)}{\tanh \sinh \left(k \sqrt{\beta_{0}}\right)}\right]+n_{1}^{2} b_{2}(z, y)+O\left(n_{1}^{3}\right),
\end{aligned}
$$

where the zero-frequency component of $b_{2}$ is

$$
\begin{aligned}
\frac{1}{L} \int_{0}^{L} d z b_{2}(z, y)= & \frac{\beta_{0}}{2}\left[\frac{k \sqrt{\beta_{0}}}{\tanh \left(k \sqrt{\beta_{0}}\right)}\left(y+\frac{1}{2}\right)-1\right. \\
& \left.+\frac{1}{\tanh ^{2}\left(k \sqrt{\beta_{0}}\right)}\right] .
\end{aligned}
$$

Third cell problem:

$$
\begin{gathered}
\mathcal{L} C=-\beta_{0}\left(2 B_{z}+A\right), \quad \mathcal{L}_{b} C=-\beta_{0} n^{\prime}(z) B, \\
\text { and } \mathcal{L}_{s} C=-\beta_{0} v^{\star 2} A,
\end{gathered}
$$

with periodic dependence in $z$ and cell average equal to zero. The solution can be expanded in powers of $n_{1}$ :

$$
\begin{aligned}
C(z, y)= & n_{1} \cos (k z)\left\{-\frac{\beta_{0}^{3 / 2}}{2} \frac{y^{2} \cosh \left(k \sqrt{\beta_{0}} y\right)}{\sinh \left(k \sqrt{\beta_{0}}\right)}\right. \\
& +\beta_{0}^{3 / 2} \frac{y \sinh \left(k \sqrt{\beta_{0}} y\right)}{\tanh \sinh \left(k \sqrt{\beta_{0}}\right)}+\frac{\cosh \left(k \sqrt{\beta_{0}} y\right)}{\sinh \left(k \sqrt{\beta_{0}}\right)} \\
& \times\left[\frac{7 \beta_{0}^{3 / 2}}{6}-\frac{\beta_{0}}{k \tanh \left(k \sqrt{\beta_{0}}\right)}-\frac{\beta_{0}^{3 / 2}}{\tanh ^{2}\left(k \sqrt{\beta_{0}}\right)}\right] \\
& \left.-\frac{\beta_{0}}{k} \frac{\sinh \left(k \sqrt{\beta_{0}} y\right)}{\sinh \left(k \sqrt{\beta_{0}}\right)}\right\}+O\left(n_{1}^{2}\right) .
\end{aligned}
$$

The effective dispersion parameter, provided in [8], is

$$
\beta^{\star}=\frac{3}{v^{\star}}\left(-\left\langle n^{\prime} C\right\rangle_{b}-v^{\star 2}\langle B\rangle_{s}\right),
$$

which, by the present results, can be expanded as

$$
\frac{\beta^{\star}}{\beta_{0}}=1+\frac{n_{1}^{2}}{2}\left[\frac{3 k \sqrt{\beta_{0}}}{\tanh ^{3}\left(k \sqrt{\beta_{0}}\right)}-\frac{11 k \sqrt{\beta_{0}}}{2 \tanh \left(k \sqrt{\beta_{0}}\right)}\right]+O\left(n_{1}^{3}\right) .
$$

\section{Fourth cell problem:}

$$
\mathcal{L} D=0, \quad \mathcal{L}_{b} D=0, \quad \mathcal{L}_{s} D=-\alpha_{0} v^{\star} A_{y y},
$$

with periodic dependence in $z$ and cell average equal to zero. The solution can be expanded in powers of $n_{1}$ :

$$
D(z, y)=-\alpha_{0} n_{1} \cos (k z) \frac{k \beta_{0} \cosh \left[k \sqrt{\beta_{0}}(y+1)\right]}{\sinh ^{2}\left(k \sqrt{\beta_{0}}\right)}+O\left(n_{1}^{2}\right) .
$$

The effective nonlinearity parameter, provided in [8], is

$$
\alpha^{\star}=\frac{1}{v^{\star}}\left(\alpha_{0}+\frac{\alpha_{0}}{3}\left\langle A_{z}^{2}\right\rangle_{s}-\frac{2}{3 v^{\star}}\left\langle n^{\prime} D\right\rangle_{b}\right),
$$

which can be expanded as

$$
\frac{\alpha^{\star}}{\alpha_{0}}=1+\frac{n_{1}^{2}}{2}\left[\left(\frac{k \sqrt{\beta_{0}}}{\sinh \left(k \sqrt{\beta_{0}}\right)}\right)^{2}+\frac{1}{2} \frac{k \sqrt{\beta_{0}}}{\tanh \left(k \sqrt{\beta_{0}}\right)}\right]+O\left(n_{1}^{3}\right) \text {. }
$$

\section{E. Optimization of the terrain-following Boussinesq system}

The expressions (25) and (27) of the effective velocity and nonlinearity parameters coincide exactly with the ones (22) and (23) derived from the multiscale expansion of the terrain-following Boussinesq system. The agreement is achieved independently of the choice of the depth parameter $y_{0}$. In order to establish a similar agreement for the dispersion parameter [compare Eqs. (24) and (26)], it is necessary to fix the parameter $y_{0} \in[0,1]$. It is remarkable that it is possible to find a value of $y_{0}$ which allows for an agreement of the dispersion parameter for any value of $\sqrt{\beta_{0}} k$, and that this value does not vary much with $\sqrt{\beta_{0}} k$, as seen in Fig. 3 .

For small $\beta_{0} k^{2}$, the agreement is achieved for $y_{0}=y_{1}:=\sqrt{2 / 3-1 / \sqrt{5}} \simeq 0.4685$.

For large $\beta_{0} k^{2}$, the agreement is achieved for $y_{0}=y_{2}:=\sqrt{1 / 3} \simeq 0.577$.

In general, the value of the depth parameter $y_{0}$ that should be chosen for equating the values of the effective dispersion parameters (24) and (26) given by the two models is between the values $y_{1}$ and $y_{2}$.

In conclusion, with the particular value of $y_{0}$ plotted in Fig. 3, the terrain-following Boussinesq system (1) and (2) and the full potential theory give the same effective $\mathrm{KdV}$ equation in the presence of a periodically varying bottom, that is, the velocity, the nonlinearity coefficient, and the dispersion coefficient are the same.

We have pointed out in the Introduction that the parameter $y_{0}$ of the Boussinesq system has been optimized in the litera- 


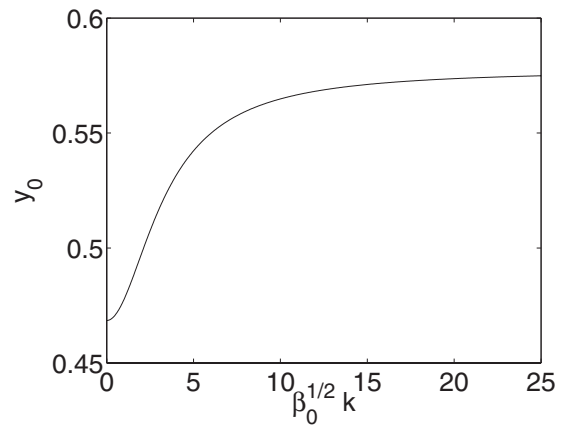

FIG. 3. The effective dispersion parameter obtained from the averaging of the terrain-following Boussinesq system is equal to the effective dispersion parameter obtained from the averaging of the Cartesian potential flow theory equations for a particular value of the depth parameter $y_{0}$ that is the function of $\sqrt{\beta_{0}} k$ plotted in this figure.

ture in order to get the best agreement between the full potential theory and the reduced Boussinesq model according to a specific criterion. In [18] a linear version of the reduced Boussinesq model was used and compared to the linear potential theory model for the multiple-scattering problem of pulse shaped waves over randomly varying topographies. In the corresponding linear flat bottom case, and working with Padé approximations for the full dispersion relation, Nwogu [11] suggested a numerical strategy for finding the optimal depth parameter $y_{0}$ regarding phase errors over a waveband of moderate wavelengths. For the linear Boussinesq model in the presence of disordered bottom topographies, Muñoz and Nachbin [18] confirmed numerically that Nwogu's best value was indeed $y_{0}=0.469$. The agreement for the result of multiply-scattered waves from both the terrain-following Boussinesq system and linear potential theory model was very good. It is remarkable that the present asymptotic analysis confirms this value from a completely different perspective, giving it a more solid foundation. Here weakly nonlinear waves over a rough topography is considered in the homogenization limit of vanishing bottom period, as opposed to linear waves in a flat bottom configuration. Based on the present analysis for rapidly varying periodic topographies, and the numerical evidence provided in [18] for rapidly varying random topographies, we can suggest one parameter value for reflection-transmission of coastal waves in the presence of fine features. Namely, the optimized terrainfollowing Boussinesq system should be considered:

$$
\begin{gathered}
M \eta_{t}+\left[\left(1+\frac{\alpha \eta}{M}\right) u\right]_{\xi}+\frac{\beta}{2}\left(\sqrt{\frac{1}{5}}-\frac{1}{3}\right)[M \eta]_{\xi \xi t}=0 \\
u_{t}+\eta_{\xi}+\alpha\left[\frac{u^{2}}{2 M^{2}}\right]_{\xi}-\frac{\beta}{2}\left(\sqrt{\frac{1}{5}}+\frac{1}{3}\right) u_{\xi \xi t}=0
\end{gathered}
$$

This optimized reduced model has the correct physical properties in the presence of both rapidly varying periodic and random topographies.

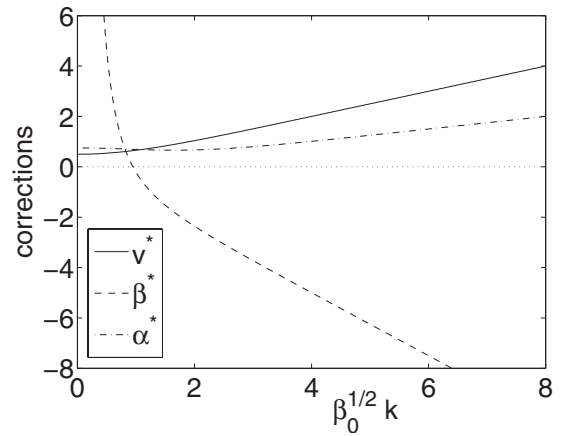

FIG. 4. Corrective terms of the coefficients of the effective KdV equation as a function of $\sqrt{\beta_{0}} k$.

\section{F. Analysis of the effective KdV equation}

In this previous sections, we have obtained explicit expressions for the coefficients of the effective $\mathrm{KdV}$ equation that are the average velocity, the nonlinearity, and dispersion coefficients. In this section, we will use these expressions in order to discuss the impact of the periodic topography on the wave propagation.

In [9] the authors obtain implicit expressions for the effective $\mathrm{KdV}$ coefficients by a multiscale expansion technique of the water wave Hamiltonian and they compute numerically these coefficients in order to study their dependence with respect to the modulation amplitude $n_{1}$. In these numerical simulations the modulation period and the depth of the channel are fixed to 1 , so that $\sqrt{\beta_{0}} k=2 \pi$ within our notation. Therefore, the dependence of the coefficients of the $\mathrm{KdV}$ equation with respect to $\sqrt{\beta_{0}} k$ is not discussed. This parameter in the original physical variables is $2 \pi$ times the ratio of the mean depth over the period of the bottom. Our explicit results, valid for small $n_{1}$, allow us to exhibit that the effective coefficients $v^{*}, \alpha^{*}$, and $\beta^{*}$ strongly depend on $\sqrt{\beta_{0}} k$. The corrective terms, which are the terms of $O\left(n_{1}^{2}\right)$ in Eq. (25)-(27), are plotted in Fig. 4. The average velocity is always reduced $\left(v^{*}<1\right)$, which was already mentioned in $[8,9]$. The nonlinearity coefficient is enhanced $\left(\alpha^{*}>\alpha_{0}\right)$. The dispersion coefficient can be reduced or enhanced, depending on the value of $\sqrt{\beta_{0}} k$. If $\sqrt{\beta_{0}} k<(\geqslant) \operatorname{arctanh}^{1 / 2}(6 / 11)$ $\simeq 0.78$, then $\beta^{*}>(\leqslant) \beta_{0}$.

When $\beta_{0} k^{2}$ is large, we have $\beta^{*} \simeq \beta_{0}-5 n_{1}^{2} \beta_{0}^{3 / 2} k / 4$ and the dispersion is reduced.

When $\beta_{0} k^{2}$ is small, we have $\beta^{*} \simeq \beta_{0}+3 n_{1}^{2} /\left(2 k^{2}\right)$ and the dispersion is enhanced. Note that, if we start with an evanescent dispersion parameter $\beta_{0}$, then the effective dispersion parameter $\beta^{*} \simeq 3 n_{1}^{2} /\left(2 k^{2}\right)$ is positive. This phenomenon has already been encountered in the literature in different contexts. In particular, apparent dispersion was seen for linear elastic waves [26] and for nonlinear elastic waves in a periodic composite [27].

\section{CONCLUSION}

In this paper we have performed a multiscale analysis of the full potential flow model for water waves over a rapidly varying periodic bottom and another one for a family of 
reduced Boussinesq models parametrized by a depth parameter. We have shown that it is possible to fit the depth parameter so that the limit $\mathrm{KdV}$ equations obtained from the two models agree. This serves to justify the use of the Boussinesq system for a wide range of bottom topography, and to strengthen the value of the depth parameter exhibited by Nwogu [11].

We have explicitly computed the coefficients of the effective $\mathrm{KdV}$ equation in the case of a small-amplitude sinusoidal topography profile and we have exhibited some inter- esting features regarding the effect of the periodic bottom on surface wave propagation. In particular, the velocity is reduced, the nonlinearity is enhanced, and the dispersion can be reduced or enhanced depending on the ratio of the mean depth over the period of the bottom.

\section{ACKNOWLEDGMENTS}

J.G. was supported by ANR, R.A.K. by CNPq, and A.N. by CNPq/Brazil under Grant No. 300368/96-8.
[1] M. W. Dingemans, Water Wave Propagation Over Uneven Bottoms (World Scientific, Singapore, 1997).

[2] C. C. Mei, The Applied Dynamics of Ocean Surface Waves (World Scientific, Singapore, 1989).

[3] J. Choi and P. A. Milewski, Stud. Appl. Math. 110, 21 (2002).

[4] C. C. Mei, J. Fluid Mech. 152, 315 (1985).

[5] V. P. Ruban, Phys. Rev. E 70, 066302 (2004).

[6] Z. Ye, Phys. Rev. E 67, 036623 (2003).

[7] M.-J. Huang, C.-H. Kuo, and Z. Ye, Phys. Rev. E 71, 011201 (2005).

[8] R. R. Rosales and G. C. Papanicolaou, Stud. Appl. Math. 68, 89 (1983).

[9] W. Craig, P. Guyenne, D. P. Nicholls, and C. Sulem, Proc. R. Soc. London, Ser. A 461, 839 (2005).

[10] A. Nachbin, SIAM J. Appl. Math. 63, 905 (2003).

[11] O. Nwogu, J. Waterway, Port, Coastal, Ocean Eng. 119, 618 (1993).

[12] P. A. Madsen and H. A. Schäffer, Philos. Trans. R. Soc. London, Ser. A 356, 3123 (1998).

[13] J. L. Bona and M. Chen, Physica D 116, 191 (1998).

[14] J. Fouque, J. Garnier, J. Muñoz Grajales, and A. Nachbin, Phys. Rev. Lett. 92, 094502 (2004).

[15] J.-P. Fouque, J. Garnier, and A. Nachbin, SIAM J. Appl. Math.
64, 1810 (2004).

[16] J. C. Muñoz Grajales and A. Nachbin, SIAM J. Appl. Math. 64, 977 (2004).

[17] J. C. Muñoz Grajales and A. Nachbin, Multiscale Model. Simul. 3, 680 (2005).

[18] J. C. Muñoz Grajales and A. Nachbin, IMA J. Appl. Math. 71, 600 (2006).

[19] J. Garnier, J. C. Muñoz Grajales, and A. Nachbin, SIAM Multiscale Model. Simul (to be published).

[20] A. Nachbin, J. Fluid Mech. 296, 353 (1992).

[21] A. Nachbin and G. Papanicolaou, J. Fluid Mech. 241, 311 (1992).

[22] C. C. Mei and Y. Li, Phys. Rev. E 70, 016302 (2004).

[23] J. R. Quintero and J. C. Muñoz Grajales, Methods Appl. Anal. 11, 15 (2004).

[24] J. M. Floryan, J. Comput. Phys. 62, 221 (1986).

[25] T. Driscoll, Schwarz-Christoffel Toolbox for MATLAB, available at http://www.math.udel.edu/ driscoll/software.

[26] F. Santosa and W. W. Symes, SIAM J. Appl. Math. 51, 984 (1991).

[27] D. H. Yong and R. J. LeVeque, SIAM J. Appl. Math. 63, 1539 (2003). 